\title{
Oral care offerings in populations of Ferlo (Senegal): The contribution of traditional dentistry ${ }^{*}$
}

\author{
Massamba Diouf $^{1 \#}$, Gilles Boetsch ${ }^{1}$, Anta Tal-Dia ${ }^{2}$, Jean Jacques Bonfil ${ }^{3}$ \\ ${ }^{1}$ CNRS-UMI 3189 “Environnement, Santé, Sociétés”, Faculté de Médecine Secteur Nord, Marseille, France \\ ${ }^{2}$ Service de Santé Publique et Médecine Préventive, Faculté de Médecine, Université Cheikh Anta Diop, Dakar, Sénégal \\ ${ }^{3}$ Service d'Epidémiologie, Faculté d'Odontologie, Marseille, France \\ Email: " dioufmass78@,yahoo.fr
}

Received 16 December 2012; revised 20 January 2013; accepted 29 January 2013

Copyright (C) 2013 Massamba Diouf et al. This is an open access article distributed under the Creative Commons Attribution License, which permits unrestricted use, distribution, and reproduction in any medium, provided the original work is properly cited.

\begin{abstract}
The aim of this study was to examine whether the offering of traditional treatments can constitute a positive contribution to integrate in an oral public health care settings. Method: This was a cross-sectional study involving $\mathbf{3 0 0}$ people living in the area of Ferlo in East Central Senegal. Selected individuals must be residents of the area over the age of 15 years. Cluster sampling was used as a recruitment method. Data collected concerned the type of oral health care used whether modern or traditional, the periodontal condition (plaque, gingival and bleeding index, attachment loss, pocket depth and CPITN). Dental status was evaluated by DMFT. The data were analyzed with $R$ software and the Student's $t$ test was used to compare the averages of indices in both groups at the threshold of $5 \%$. A co-variance analysis was allowed in order to determine associations between some indices and therapy type in multivariate approach. Results: Averages of dental and periodontal indices were globally higher in people using modern therapies. DMFT, CPITN and gingival index increased significantly when the type of therapy was modern after adjustment on the plaque index and age. Conclusion: The traditional dentistry could constitute a positive contribution to a public health care, especially in a context of developing country. However, this practice should be guided and validated by a research-based evidence to allow better use of the therapeutic processes, and promote the health of populations.
\end{abstract}

Keywords: Oral Care Supply; Senegal; Traditional Dentistry

${ }^{*}$ Conflict of interest: there is no conflict of interest about this manuscript.

${ }^{\#}$ Corresponding author.

\section{INTRODUCTION}

Traditional dentistry is by analogy to traditional medicine, the set of all knowledge and practices; explained or not; used to diagnose, prevent or eliminate a physical, mental or social imbalance in relation to teeth; taking into account experiences lived or transmitted, metaphysical forces and nature [1]. It is an important part of healthcare offers proposed to the populations of developing countries particularly those in rural areas due to the expensive cost of care and the lack of qualified dental staff. In Africa, the dentist population ratio is about 1 for 150,000 [2], which is still far from the standards advocated by the World Health Organization in support of oral health (1 dentist to ten thousand inhabitants). In Senegal, the ratio is a dentist for 32,500 inhabitants [3], with larger disparities in rural areas. In the rural areas of Ferlo, there are few dentists. The only operational dental surgery unit is 100 kilometers far with a rugged terrain making it a difficult point to access for the population. This testifies for the inadequate coverage of dental pathologies by modern medicine that could force people to resolve to other treatment options and other practitioners. According to the WHO, almost $80 \%$ Africans rely on this practice as first-line [4]. Some countries such as Mali, Ghana, Burkina Faso and Ivory Cost have developed strategic health policies to promote these forms of treatment [5]. Many isolated studies have called the empirical value of certain plants in Odontostomatology [6,7]. However, the scientific basis of these results remains to be elucidated. In addition, low dental or periodontal clinical parameters in patients treated by these traditional practitioners are rare. For this reason, it is logical to ascertain whether these therapies allow an improvement of oral health especially for people in rural areas, where access to modern oral care is often difficult.

The objective of this work is to examine whether the 
offer of traditional treatments can constitute a positive addition to integrate into oral public health care for the rural population of Senegal within certain limits.

\section{METHOD}

It was a cross-sectional study involved the population living in Ferlo area in East Central Senegal. Ferlo is in the perimeter of the Senegal's great green wall. Its density is an average of 34 inhabitants per $\mathrm{Km}^{2}$.

\subsection{Selection Criteria}

To be selected in the study, individuals had to be over the age of 15 years and reside in the area. These individuals should also have a general health status that allows oral clinical examination and agree to participate in the study.

\subsection{The Sample Size}

The sample size was determined by the formula of Schwartz:

$\mathrm{n}=(\varepsilon \alpha)^{2} \mathrm{pq} / \mathrm{I}^{2}$ which can be used in cross-sectional studies where $\varepsilon=\mathrm{z}$ score $=1.96 . \alpha=$ error risk $=0.05 ; \mathrm{p}$ $=$ prevalence of health phenomenon (dental caries) found after pilot investigation in 15 individuals randomly selected on the spot. This prevalence was $89 \% ; \mathrm{q}=1-\mathrm{p}=$ $11 \% ; \mathrm{I}=$ precision $=5 \%$. These settings enabled to have a size of 150 individuals. Considering an effect of cluster sampling, this size was multiplied by 2 . Finally 300 people were selected for the survey.

\subsection{Sampling}

Cluster sampling was used. Out of a total of 20 villages, after the first random sampling, ten primary clusters of villages were selected. Afterwards, a secondary random sampling within each village allowed us to choose the houses. The choice of houses was made according to the survey method of the World Health Organization in which we chose the houses by moving closer and closer to the house firstly selected at random. Once the house is chosen, all the people living in and who met the selection criteria were examined. A maximum of 30 people was set by village making a total of 300 for the ten selected villages

\subsection{Collection Procedure and Variables}

The data were collected with the WHO 1997 questionnaire, modified and adapted to the context of Ferlo. A group of 5 Investigators made up by 4 dentists assisted by a PhD student in dentistry. All passed a standardized training to avoid classification bias. The recruitment took place every day from April to May 2010.

The epidemiological information collected concerned:
- The treatment of choice in case of oral problem (modern or traditional). Type of treatment: herbal medicine and/or incantatory formulas for traditional therapy, or visit to the dentist for modern treatments.

- The dental status was assessed by the DMFT.

- Periodontal status was evaluated by the plaque, gingival, bleeding indices and loss of clinical attachment pocket depth. The needs of periodontal treatment (CPITN) were also used.

\subsection{Clinical Observation}

DMFT index translated the number of decayed permanent teeth (D), missing (M) and filled teeth (F) of the study population.

The average DMFT index was given by the ratio between the total number of teeth DMF and the number of persons examined.

Periodontal index were measured using the recommendations of Martin and Bercy [8].

We had 20 examination sets, each includes: 6 probe, 17 probe, Williams probe, mirror; tweezers; gloves, masks and fields of work.

\subsection{Ethical Considerations}

Prior to the examination of each individual, free and informed consent was first solicited.

After the clinical examination, each person was offered a session of oral hygiene and a package containing a toothbrush and toothpaste. Information from each individual was treated confidentially.

\subsection{Analysis of the Data}

The software Epi info 3.4.5 was used for data processing. Descriptive data was presented in tables for better understanding of differences, in indices studied, between people who used modern therapy with those who used traditional therapy after stratification. The Student's t-test was used at the threshold of 5\% in the bivariate analysis.

Secondly, multivariate analysis of covariance was developed for indices that were significantly associated with the type of therapy (modern or traditional). An adjustment on the plaque index and age was made. The mean square and the sum of the squares were determined at the threshold of $5 \%$.

\section{RESULTS}

More than $50 \%$ of the sample were male, $51.7 \%$ were aged between 15 - 35 years old.

More than $70 \%$ of individuals in our sample (71.3\%) used traditional therapies versus $28.7 \%$ used modern care.

The mean plaque index was high in people using 
modern dentistry, however, Student's test did not show a significant difference between the two groups. Meanwhile, the mean gingival index was higher among those attending the dentist with a significant difference $(\mathrm{p}=$ 0.004).

Gingival bleeding and loss of clinical attachment appeared less important in people using traditional treatments without significant statistical difference.

Periodontal pockets were slightly less deep in people using traditional care yet the difference was not significant at the level of 5\% (t-bilateral). It should be noted that the average was 2.2 for therapies of modern care versus 2.07 for the traditional care.

The needs for periodontal treatments were higher for persons who used modern care with a statistically significant difference $(\mathrm{p}=0.04)$.

For dental status average, DMFT, was most important in people using modern care $(p=0$. 007). The extreme value of the DMFT (30) was found among those who consumed modern care, moreover, it should be noted that the average DMFT in modern care group was 6.02 versus 4.3 for the traditional treatment group.

The DMFT increased significantly when the type of therapy was modern even after adjustment for and age and plaque indices. It was the same tendency with CPITN and gingival index.

\section{DISCUSSION}

This study found that rural populations particularly those of Ferlo mostly use traditional therapy when they suffer from dental problems. Several dental and periodontal clinical parameters assessed by different indices, were overall better in people using modern care. However, the collection of the variable "type of therapy" was made on the basis of declaration of people at time of the survey. This might have overestimated or underestimated the link found between therapy type and others variables. The ideal would be to collect this variable from their medical or dental record in health care settings. Given the low coverage of oral health professionals, the absence of dental care structures in rural areas and the isolation of these villages, which are, often, difficult to access, it may seem fair to measure the variable "type of therapy used" by simple declaration.

If treatment of a disease is currently done by processed drugs of complex formations, the treatment option in many countries still involves ancestral therapies based essentially on the use of plants. Besides, the World Health Organization has recognized that the African people do not have access to the so-called modern drugs and that they resolve only to this form of practice for treatment [4]. Sangare et al. in Côte d'Ivoire [5] reported that only $20 \%$ of the inhabitants of the town use this therapy as first-line. This percentage seems small in comparison to the proportion of $70 \%$ that we have found. This gap can be explained by difference between urban and rural areas. In the otherwise, the illiteracy of population could be explained this attitude because more than $3 / 4$ of people $(78 \%)$ were not in school (Table 1 ).

Several therapies are used traditional care. They can be incantatory or magico-religious forms, manual techniques or herbal medicine.

Higher average DMFT among individuals who use modern care structures is explained by its scarcity and its geographical and financial inaccessibility. Knowing that the modern care settings are extremely far people tend to wait and not to go unless it is very serious and they can't wait more. If people would wait to this point, logically, this would result in worsening of their conditions. Traditional therapies, for those who use them, may stop or slow down the disease process, which would explain the lower average of the DMFT in this group (Table 2). It is likewise for the periodontal parameters. Campus et al. [9] have highlighted the benefits of Magnolia, this scented White flowering plant in the form of large tulips throughout the summer, for dental plaque and bleeding in a randomized double blind clinical trail.

The covariance analysis performed showed that the DMF index, the CPITN, gingival index and the pocket depth increased significantly among those using modern therapy even after adjustment on age and plaque index (Table 3). Even though modern care is more effective

Table 1. Distribution of sociodemographic variables.

\begin{tabular}{cccc}
\hline Variables & & Number & Percentage (\%) \\
\hline \multirow{2}{*}{ Sex } & Male & 165 & 55 \\
& Female & 135 & 45 \\
\multirow{2}{*}{ Age } & $15-35$ years old & 155 & 51.7 \\
& $>35$ years old & 145 & 48.3 \\
\multirow{2}{*}{ Schooling } & Yes & 66 & 22 \\
& No & 234 & 78 \\
\hline
\end{tabular}

Table 2. Relationship between the means of periodontal and dental indices and type of therapy (traditional or modern) used.

\begin{tabular}{ccccc}
\hline \multirow{2}{*}{ Variables } & $\begin{array}{c}\text { Traditional } \\
\text { therapy }\end{array}$ & $\begin{array}{c}\text { Modern } \\
\text { therapy }\end{array}$ & \\
\hline & Plaque & 1.61 & 1.64 & 0.33 \\
& Gingival & 1.38 & 1.53 & 0.004 \\
Periodontal & Bleeding & 1.54 & 1.61 & 0.31 \\
status & Attachment loss & 2.62 & 2.63 & 0.89 \\
& Pocket depth & 2.07 & 2.2 & 0.07 \\
& CPITN & 1.39 & 1.55 & 0.004 \\
\hline \multirow{2}{*}{ Dental status } & DMFT & 4.29 & 6.01 & 0.007 \\
\hline
\end{tabular}


Table 3. Co-variance of DMFT, CPITN, gingival index, pockets depth and the type of therapy used after adjustment for age and plaque index.

\begin{tabular}{ccccccc}
\hline & Variation & df & Sum Sq & Mean Sq & F & p-value \\
\hline \multirow{3}{*}{ DMFT } & Therapy type & 1 & 132.4 & 132.4 & 7.77 & $0.005^{*}$ \\
& Plaque index & 1 & 57.7 & 57.7 & 3.39 & 0.066 \\
& Age & 1 & 2.3 & 2.3 & 0.13 & 0.712 \\
\multirow{3}{*}{ CPITN } & Therapy type & 1 & 12,442 & 12,442 & 10.2 & $0.001^{*}$ \\
& Plaque index & 1 & 91,165 & 91,165 & 74.8 & $<0.001^{*}$ \\
& Age & 1 & 7226 & 7226 & 5.9 & $0.015^{*}$ \\
\multirow{3}{*}{ Gingival index } & Therapy type & 1 & 10,162 & 10,162 & 18.3 & $<0.001^{*}$ \\
& Plaque index & 1 & 149,869 & 149,869 & 270.6 & $<0.001^{*}$ \\
& Age & 1 & 2097 & 2097 & 3.7 & 0.05293 \\
& Therapy type & 1 & 8291 & 8291 & 2.7 & 0.098 \\
& Plaque index & 1 & 19,136 & 19,136 & 6.3 & $0.012^{*}$ \\
\hline
\end{tabular}

${ }^{*}=$ significant.

for some diseases because of manual dexterity and advanced technology, this study has also showed the relatively beneficial character of traditional therapies. The cranberry polyphenols, a shrub that grows in the bogs of cold regions, according to Blackburn and attic in Canada [10] play a preventive role against dental caries and periodontal diseases. On one hand, they inhibit the production of organic acid and biofilm formation of cariogenic bacteria; and they reduce the inflammatory response and the proteolytic enzymes activity participating in bone Lysis in periodontal disease on the other.

\section{CONCLUSION}

Traditional dentistry is increasingly used in rural areas of Africa and in Senegal particularly. It could be a positive contribution in a public health care, especially, in a rural area. However, this practice should be organized and guided by a research-based evidence to enable a better use of the therapeutic processes, and promote the health of the population.

\section{ACKNOWLEDGEMENTS}

We thank the nurse of Ferlo land for support and providing access to the participants.

\section{REFERENCES}

[1] World Health Organization (1978) Traditional medicine and its role in the development of the health services in Africa. Technical Specifications, Geneva, 1-114.

[2] World Health Organization (2007) Oral health. Genève,
1-318.

[3] World Health Organization (2010) The health oral. Geneva, 1-6.

http://www.slideshare.net/zakariadds/la-sante-bucco-dent aire

[4] World Health Organization (2000) General methodological principles for research and assessment related to traditional medicine. Genève, 1-87. http://rds.refer.sn/IMG/pdf/CODEBIBLIO.pdf

[5] Sangaré, A., Samba, M., Guinan, J.C., Bakayoko-Ly, R. and Bourgeois, D. (2011) Traditional practices and exploitation of medicinal plants in odontostomatology in Ivory Coast. Tropical Odontostomatology, 1, 21-26.

[6] Diouf, M., Diouf, A., Cisse, D., Touré, B., Lo, C.M.M. and Faye, D. (2011) Medicinal plants in Senegal and oral diseases: Use and interest. Tropical Odontostomatology, 3, 11-16.

[7] Diallo, B. and Diouf, A. (2000) Study of the analgesic activity of Pilostigma reticulatum (Nguiguis). Tropical Odontostomatology, 92, 5-11.

[8] Martin, A. and Bercy, P. (2002) Review of currently used periodontal indexes. Belgian Journal of Dentistry, 3, 215243

[9] Campus, G., Cagetti, M.G., Cocco, F., Sale, S., Sacco, G., Strohmenger, L. and Lingström, P. (2011) Effect of a sugar-free chewing gum containing magnolia bark extract on different variables related to caries and gingivitis: A randomized controlled intervention trial. Caries Research, 4, 393-399. doi:10.1159/000330234

[10] Bonifait, L. and Grenier, D. (2010) Cranberry polyphenols: Potential benefits for dental caries and periodontal disease. Journal of Canadian Dental Association, 76, 123-130. 